\title{
Teaching the Basics: Development and Validation of a Distal Radius Reduction and Casting Model
}

\author{
Mark A. Seeley MD, Peter D. Fabricant MD, MPH, J. Todd R. Lawrence MD, PhD
}

Received: 3 November 2016/Accepted: 24 March 2017/Published online: 3 April 2017

(C) The Association of Bone and Joint Surgeons (B) 2017

\begin{abstract}
Background Approximately one-third of reduced pediatric distal radius fractures redisplace, resulting in further treatment. Two major modifiable risk factors for loss of reduction are reduction adequacy and cast quality. Closed reduction and immobilization of distal radius fractures is an Accreditation Council for Graduate Medical Education residency milestone. Teaching and assessing competency could be improved with a life-like simulation training tool. Questions/purposes Our goal was to develop and validate a realistic distal radius fracture reduction and casting
\end{abstract}

The institution of one or more of the authors has received, during the study period, funding from Sawbones Inc through an OMeGa Core Innovation Grant \#001046 (JTRL).

All ICMJE Conflict of Interest Forms for authors and Clinical Orthopaedics and Related Research ${ }^{\mathbb{R}}$ editors and board members are on file with the publication and can be viewed on request.

Children's Hospital of Philadelphia approved the human protocol for this investigation, and the authors certify that all investigations were conducted in conformity with ethical principles of research, and that informed consent for participation in the study was obtained. This work was performed at Children's Hospital of Philadelphia, Philadelphia, PA, USA.

Electronic supplementary material The online version of this article (doi:10.1007/s11999-017-5336-3) contains supplementary material, which is available to authorized users.

M. A. Seeley ( $\square)$

Geisinger Medical Center, 100 N Academy Avenue, Danville, PA 17821, USA

e-mail: mseeley1@geisinger.edu; mseeley21@gmail.com

P. D. Fabricant

Hospital for Special Surgery, New York, NY, USA

J. T. R. Lawrence

Children's Hospital of Philadelphia, Philadelphia, PA, USA simulator as determined by (1) a questionnaire regarding the "realism" of the model and (2) the quantitative assessments of reduction time, residual angulation, and displacement.

Methods A distal radius fracture model was created with radiopaque bony segments and articulating elbows and shoulders. Simulated periosteum and internal deforming forces required proper reduction and casting techniques to achieve and maintain reduction. The forces required were estimated through an iterative process through feedback from experienced clinicians. Embedded monofilaments allowed for quantitative assessment of residual displacement and angulation through the use of fluoroscopy. Subjects were asked to perform closed reduction and apply a long arm fiberglass cast. Primary performance variables assessed included reduction time, residual angulation, and displacement. Secondary performance variables consisted of number of fluoroscopic images, casting time, and cast index (defined as the ratio of the internal width of the forearm cast in the sagittal plane to the internal width in the coronal plane at the fracture site). Subject grading was performed by two blinded reviewers. Interrater reliability was nearly perfect across all measurements (intraclass correlation coefficient range, 0.94-0.99), thus disagreements in measurements were handled by averaging the assessed values. After completion the participants answered a Likert-based questionnaire regarding the realism of simulation. Eighteen participants consented to participate in the study (eight attending pediatric orthopaedic surgeons, six junior residents, four senior residents). The performances of junior residents (Postgraduate Year [PGY] 1-2), senior residents (PGY 3-5), and attending surgeons were compared using one-way ANOVA with Tukey's-adjusted pairwise comparisons. 
Results The majority of participants (15 of 18) felt that the model looked, felt, and moved like a human forearm. All participants strongly agreed that the model taught the basic steps of fracture reduction and should be implemented in orthopaedic training. Attending surgeons reduced fractures in less time than junior residents $(60 \pm$ 27 seconds versus $460 \pm 62$ seconds; mean difference, 400 seconds; 95\% CI, 335-465 seconds; $\mathrm{p}<0.001)$. Residual angulation was greater for junior residents when compared with attending surgeons on $\mathrm{AP}\left(7^{\circ} \pm 5^{\circ}\right.$ versus $0.7^{\circ} \pm 0.9^{\circ}$; mean difference, $\left.6.3^{\circ} ; 95 \% \mathrm{CI}, 3^{\circ}-11^{\circ} ; \mathrm{p}=0.003\right)$ and lateral $\left(27^{\circ} \pm 7^{\circ}\right.$ versus $7^{\circ} \pm 5^{\circ}$; mean difference, $20^{\circ}$; $\left.95 \% \mathrm{CI}, 13^{\circ}-27^{\circ} ; \mathrm{p}=0.001\right)$ radiographs. Similarly, residual displacement was greater for junior residents than either senior residents (mean difference, $16 \mathrm{~mm}$; $95 \% \mathrm{CI}$, 2-34 mm; $\mathrm{p}=0.05$ ) or attending surgeons (mean difference, $15 \mathrm{~mm}$; 95\% CI, 3-27 mm; $\mathrm{p}=0.02$ ) on lateral images. There were no differences identified in secondary performance variables (number of fluoroscopic images, casting time, and cast index) between groups.

Conclusions This is the first distal radius fracture reduction model to incorporate an elbow and shoulder and allow quantitative assessment of the fracture reduction. This simulator may be useful in an orthopaedic resident training program to help them reach a defined minimum level of competency. This simulator also could easily be integrated in other accreditation and training programs, including emergency medicine.

Level of Evidence Level II, therapeutic study.

\section{Introduction}

Nonoperative management through closed reduction and immobilization is the mainstay of treatment for the majority of distal radius fractures in the pediatric and adolescent population $[3,4,14,16,17,19,21]$. Unfortunately, approximately $1 / 3$ of the reduced distal radius fractures will redisplace, with approximately $7 \%$ requiring additional treatment $[2-4,12,18-20,22]$. The consequences of redisplacement are profound, yet the majority of the front-line treatment of these fractures is left with some of the most inexperienced providers. Distal radius fractures requiring manipulation and immobilization require not only sound reduction techniques but also sound casting fundamentals. In the past, fracture reduction and cast molding have been taught didactically covering general concepts and demonstrating basic techniques. After the institution of work-hour limitations and with increasing concerns for patient safety, orthopaedic training is facing a paradigm shift in how clinical skills are being taught. Teaching methods are favoring simulated hands-on training rather than didactic lectures because it provides an opportunity for repetition and objective feedback [1, 7, 23].

Loss of reduction can be related to the fracture pattern. However, for simple fracture patterns, the major risk factors for redisplacement appear to be modifiable factors, namely inadequate reduction, poor cast molding, and inexperience of the provider [2, 4, 12, 18, 19, 21, 24, 25]. Previous authors have developed distal radius reduction models [9, 13, 15]. These models are anchored at the forearm through a table clamp and focus on manipulation of the fracture fragments [9, 13, 15]. Although the face validity (realism of simulation) has been rated high among participants in noncomparative studies [9, 13], some of these models lack real-time objective feedback making quality assessment difficult and cannot simulate all aspects of the care delivery process likely to be encountered by the resident. Fracture reduction and cast placement are core orthopaedic principles. Teaching and assessing technical competency of these skills while minimizing patient risk are challenging but essential components of residency training [10]. This task has been hampered by the lack of a training tool.

The purpose of this study was to develop and validate a simple yet realistic distal radius fracture reduction and casting simulator as determined by (1) a questionnaire regarding the "realism" of the model, and (2) the quantitative assessments of reduction time, residual angulation, and displacement.

\section{Materials and Methods}

This prospective observational study was reviewed and approved by the institutional review board of Children's Hospital of Philadelphia and consent was obtained from all participants. The study was performed at a single institution during a course of 4 months. All residents from the single home institution on their pediatric orthopaedic rotation participated. All participants completed a reduction questionnaire with only seven participants having prior simulation training in other procedures. The life-like model of the upper extremity was developed by one of the authors (JTRL) and the Surgical Simulation Program at Children's Hospital of Philadelphia with engineering assistance and model production performed by Pacific Research Laboratories Inc (Sawbones ${ }^{\circledR}$, Vashon Island, WA, USA). Pilot testing was performed with a separate cohort to refine all aspects of model design, materials, and tension before study initiation. The model was designed to mimic the usual periosteal restraints and deforming muscular forces at work in a simple transverse distal radius fracture. Multiple prototypes were trialed with tension and location of the straps being varied to balance the qualitatively desired 
biomechanical effect with the engineering and material limitations. Iterative feedback on design and tensioning was received from a volunteer panel of experienced orthopaedic surgeons who provided a qualitatively based consensus final decision on the design.

The internal skeleton of the model was constructed of synthetic radius and ulna bones mounted to an articulating hinge to simulate elbow flexion and extension. The bones were coated with radiopaque paint to allow for observation with standard fluoroscopy. The arm was attached to a table clamp that allowed three planes of shoulder motion (flexion-extension, abduction-adduction, and internal-external rotation) and simulated the position of a patient who has been placed at the edge of the table with the shoulder just off the edge of the bed (Fig. 1).

The internal model construction included a nylon strap secured to the dorsal aspect of the distal radius to simulate periosteum. This strapping continued across the wrist to simulate the dorsal and volar wrist ligaments (Fig. 2). Attachment points on the nylon strap were fashioned to simulate apex-volar fracture displacement and block reduction if the reduction was not adequately performed. The reduction required sequential steps: recreation of dorsal angulation followed by length acquisition, then displacement correction, and finally angulation correction. An additional dynamically tensioned nylon cord was attached to the dorsal radial aspect of the distal radial fragment to simulate deforming forces (Fig. 2). The inflection point of this dynamic displacing force was designed to allow for stable reduction with little force if the fracture was reduced anatomically. However, release of this minimal force on an anatomically reduced fracture caused fracture angulation and displacement (Fig. 3). Monofilament radiopaque markers were embedded in the distal radius. A simple extraarticular transverse distal radius fracture was created using an extrafine rotary bit. The radiopaque markers allowed for quantification of displacement and angulation during assessment (Fig. 4). The model was covered with a polyurethane foam and siliconebased skin to allow for normal cast application (Figs. 1,3). The bones and overall size of the limb were sized to be that of the average late adolescent with distal-forearm and midforearm circumferences measuring $18 \mathrm{~cm}$ and $22 \mathrm{~cm}$, respectively.

Participants (junior residents [postgraduate year (PGY) 1-2], senior residents (PGY 3-5), and attending surgeons) were recruited for the study and asked to reduce and cast a $100 \%$ displaced and angulated distal radius fracture on the model as if they were in the emergency department or operating room setting. Attending surgeons were eligible if they were pediatric fellowship trained. Eighteen participants consented to participate in the study (eight attending pediatric orthopaedic surgeons, six junior orthopaedic residents, four senior orthopaedic residents). For the purposes of the current study, the participants were not given parameters of acceptable reductions nor were they told when they had an acceptable reduction. They simply were asked to do the best that they could with a single "attempt." Participants were allowed as much time as needed to obtain the reduction and place a cast. Final assessment was performed after application of the fiberglass cast. The simulation took place in the operating room setting
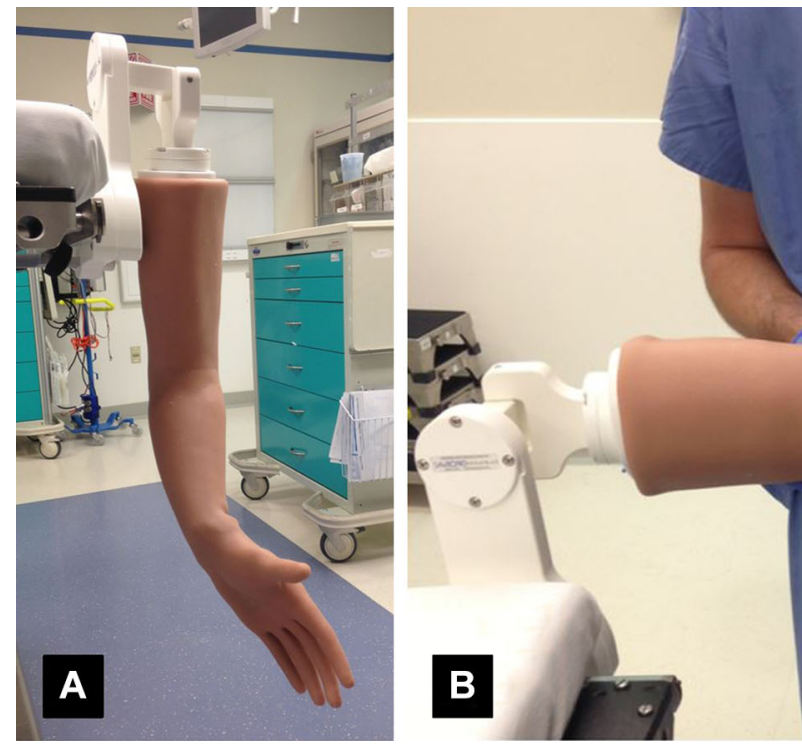

Fig. 1A-B The distal radius reduction model consisted of a synthetic radius and ulna mounted to $(\mathbf{A})$ an articulating hinge to simulate an elbow allowing flexion and extension. The simulated (B) shoulder attached to a table clamp allowed three planes of movement (flexionextension, internal-external rotation, and abduction-adduction). 
equipped for surgical simulations with the appropriate personnel (C-arm operator) and supplies (large C-arm, protective lead garments, traction/finger traps if desired,
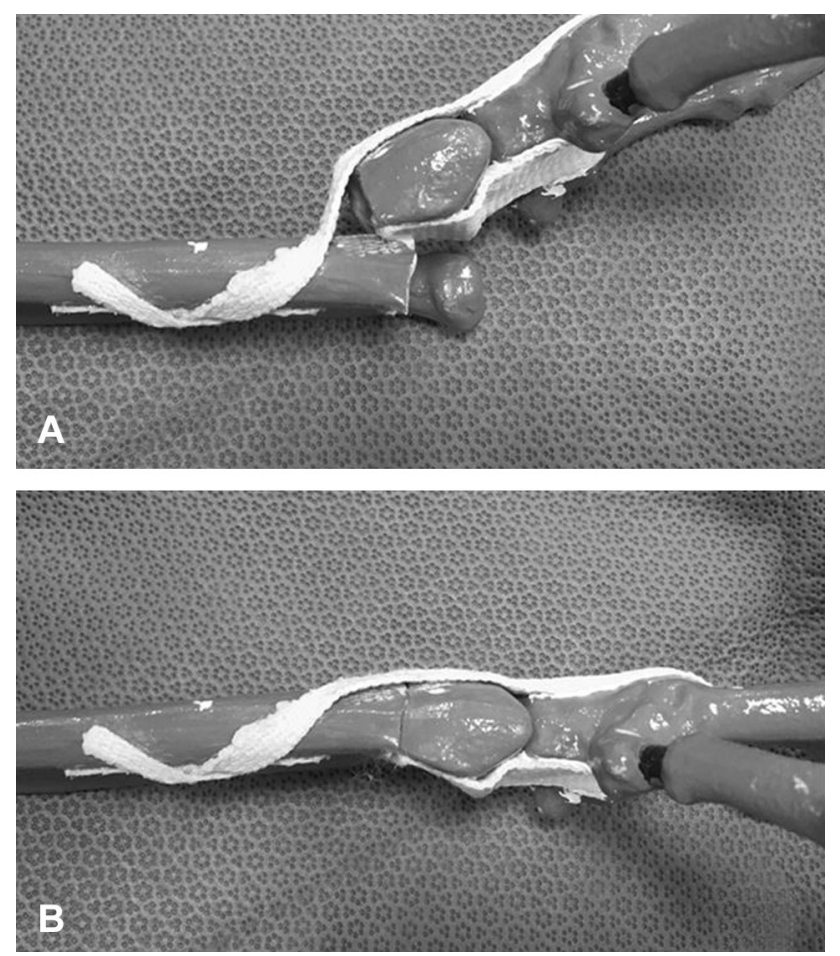

Fig. 2A-B (A) The internal model construction included a nylon strap secured to the dorsal aspect of the distal radius to simulate periosteum. (B) This strapping continued across the wrist to simulate the dorsal and volar wrist ligaments. Attachment points on the nylon strap were fashioned to simulate apex-volar fracture displacement and block reduction if the reduction was not adequately performed. and casting materials) as would be expected for delivering usual clinical care. Primary performance variables included total reduction time, residual angulation, and displacement. Secondary variables consisted of number of fluoroscopic images required to obtain reduction, casting time, and cast index. Cast index was defined as the ratio of the internal width of the forearm cast in the sagittal plane to the internal width in the coronal plane at the fracture site.

At the conclusion of the simulation, standardized AP and lateral fluoroscopic views were obtained. Measurements of angulation and displacement were made on the best AP and lateral images after the cast had been applied. The fluoroscopy machine prevented measurements in millimeters; therefore, all measurements were made as a proportion of the voxels measured at the designated area normalized to the width of the radial shaft (Fig. 4). The radiopaque monofilaments allowed for displacement and angulation to be judged at the level of the fracture site. Displacement was measured as a percentage of displacement in relation to the distal fragment piece on the AP image. On the lateral image, lines were subtended from the monofilaments and the area between the lines at the level of the fracture site was measured. Measurement of residual angulation at the fracture site was performed on AP and lateral images and reported as absolute values (Fig. 4).

A questionnaire modeled after that of Egan et al. [9] was constructed to assess participant opinion of model characteristics, which included appearance, tactile feel, and ability to manipulate (Appendix 1. Supplemental material is available with the online version of $C O R R^{\mathbb{R}}$.). These data were collected using a five-level Likert scale with the scaling format ranging from: strongly disagree (1);
Fig. 3A-B (A) The simulated periosteum was fashioned to redisplace if inadequate traction was applied before attempting reduction of the dorsal angulation, an incorrect reduction maneuver, or improper casting technique was performed. (B) Proper reduction technique was required to reduce the fracture and maintain the reduction during the simulation.
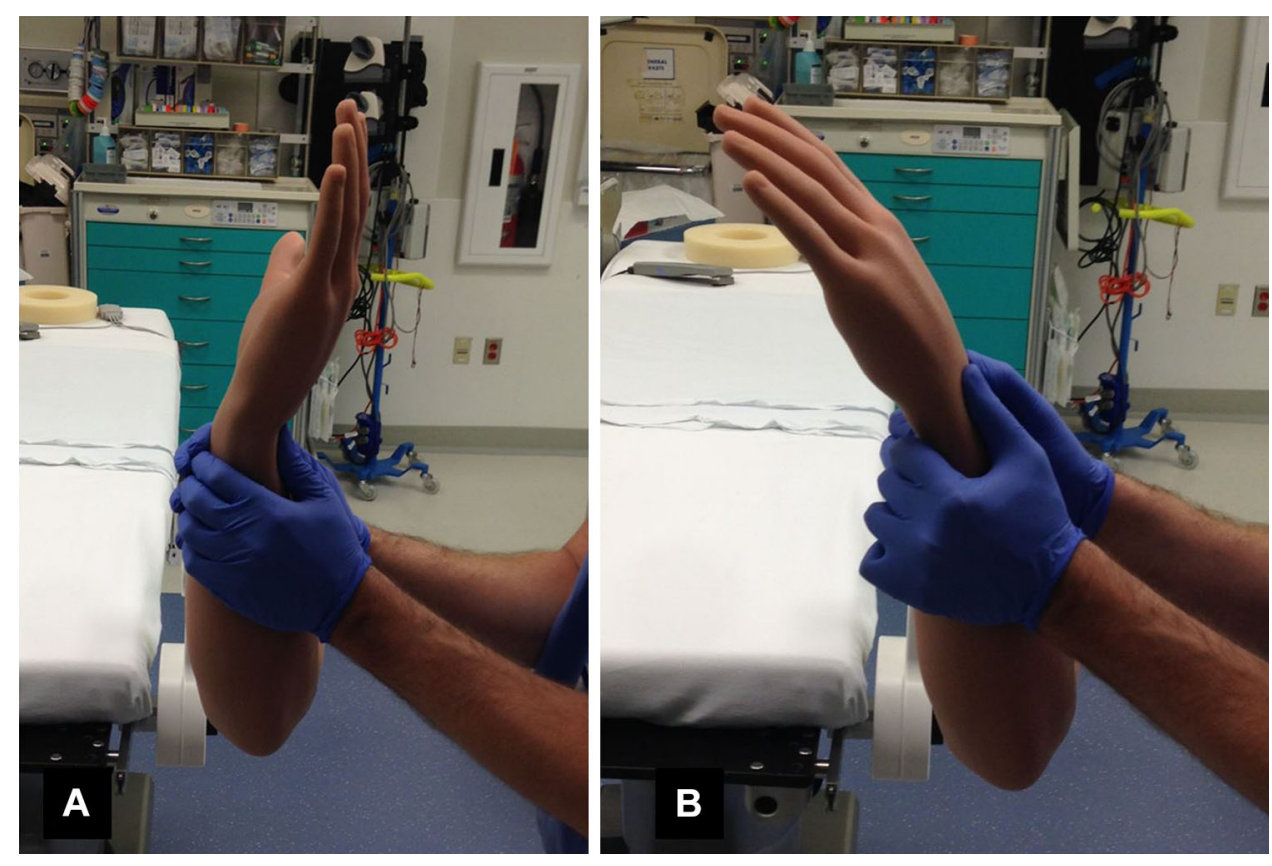
Fig. 4A-B (A) Lateral and (B) AP fluoroscopic images show postreduction and casting. Radiopaque monofilaments allow for accurate assessment of angulation and displacement.
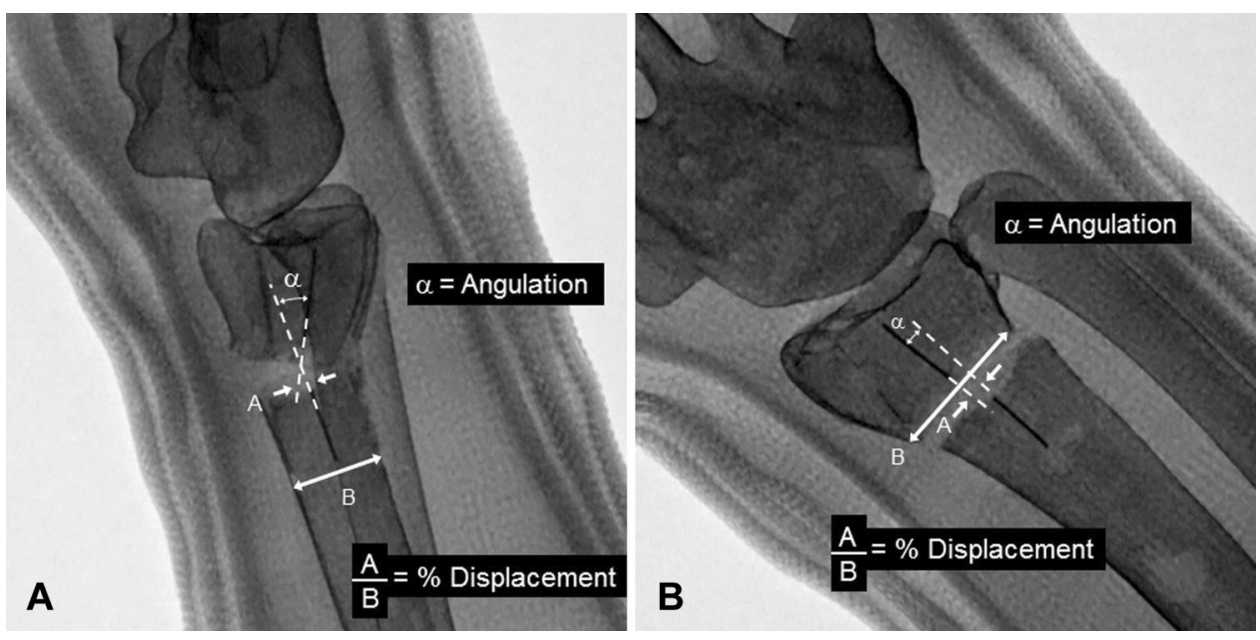

disagree (2); neither agree nor disagree (3); agree (4); and strongly agree (5). Participants also were asked to rate their experience and overall usefulness of the simulation.

\section{Statistical Analysis}

Descriptive summaries and statistical testing was performed using IBM SPSS Statistics Version 22.0 software (IBM Corporation, Armonk, NY, USA). Construct validity was achieved by stratifying the results of the primary and secondary performance variables by level of training. The performances of junior residents, senior residents, and attending surgeons were compared with each other using one-way ANOVA with Tukey's adjusted pairwise comparisons. Subject grading was performed by two blinded reviewers (MAS, JTL) and interrater reliability was assessed using the intraclass correlation coefficient (ICC [2, 1]). Disagreements in measurements were handled by averaging the values. Probability values less than 0.05 were considered statistically significant, and all comparative analyses were two-tailed.

\section{Results}

The majority of participants (15 of 18) thought that the model looked, felt, and moved like a human forearm. All participants agreed that the fracture model provided tactile feedback during the reduction (Appendix 1. Supplemental material is available with the online version of $C O R R^{\circledR}$.). In addition, all participants strongly agreed that the model taught the basic steps of fracture reduction and should be implemented in orthopaedic training (Appendix 1. Supplemental material is available with the online version of $C O R R^{\mathbb{R}}$.).

Attending surgeons and senior residents completed the fracture reduction faster and more accurately than junior residents, supporting the construct validity of this model. Attending surgeons were faster than junior residents with reduction before casting ( $60 \pm 27$ seconds versus $460 \pm 62$ seconds; mean difference, 400 seconds; 95\% CI, 335-465 seconds; $\mathrm{p}<0.001)$. Residual angulation was greater for junior residents when compared with attending surgeons on AP $\left(7^{\circ} \pm 5^{\circ}\right.$ versus $0.7^{\circ} \pm 0.9^{\circ}$, respectively; mean difference, $\left.6.3^{\circ} ; 95 \% \mathrm{CI}, 3^{\circ}-11^{\circ} ; \mathrm{p}=0.003\right)$ and lateral $\left(27^{\circ}\right.$ $\pm 7^{\circ}$ versus $7^{\circ} \pm 5^{\circ}$, respectively; mean difference, $20^{\circ}$; $95 \% \mathrm{CI}, 13^{\circ}-27^{\circ} ; \mathrm{p}=0.001$ ) radiographs (Table 1 ). Greater residual angulation also was more likely for junior residents than for senior residents on $\mathrm{AP}\left(7^{\circ} \pm 5^{\circ}\right.$ versus $2.1^{\circ} \pm 1.3^{\circ}$; mean difference, $5^{\circ} ; 95 \% \mathrm{CI}, 0.2^{\circ}-11^{\circ} ; \mathrm{p}=$ $0.05)$ and lateral $\left(27^{\circ} \pm 7^{\circ}\right.$ versus $11^{\circ} \pm 4^{\circ}$; mean difference, $\left.16^{\circ} ; 95 \% \mathrm{CI}, 7^{\circ}-26^{\circ} ; \mathrm{p}=0.001\right)$ radiographs (Table 1). In addition, greater displacement was more likely with junior residents than either senior residents (mean difference, $16 \mathrm{~mm} ; 95 \% \mathrm{CI}, 2-34 \mathrm{~mm} ; \mathrm{p}=0.05$ ) or attending surgeons (mean difference, $15 \mathrm{~mm}$; 95\% CI, 3$27 \mathrm{~mm} ; \mathrm{p}=0.02$ ) on lateral images (Table 1).

Analysis of secondary performance variables revealed that junior residents required a longer time to place the cast after the reduction than attending surgeons $(803 \pm 152$ seconds versus $451 \pm 125$ seconds; mean difference, 352 seconds; 95\% CI, 106-599 seconds; $\mathrm{p}=0.01$ ). However, no difference in cast index was identified between groups (junior residents, $0.76 \pm 0.08$; senior residents, $0.70 \pm$ 0.04 ; attending surgeons, $0.67 \pm 0.09$; ANOVA $\mathrm{p}=0.2$ ). In the study cohort, there was no difference in the quantity of fluoroscopic images taken between junior residents and attending surgeons $(\mathrm{p}=0.38)$.

\section{Discussion}

Currently, the majority of distal radius reduction models use fractured synthetic bones; however, the lack of soft 
Table 1. Residual angulation and displacement stratified by year in training

\begin{tabular}{|c|c|c|c|c|c|c|}
\hline Performance variables & Junior resident & Senior resident & Attending surgeon & $\mathrm{p}$ value, ANOVA & $\mathrm{p}$ value, comparisons & Tukey-adjusted pairwise \\
\hline \multicolumn{7}{|c|}{ Residual angulation (degrees) } \\
\hline $\mathrm{AP}$ & $7^{\circ} \pm 5^{\circ}$ & $2^{\circ} \pm 1^{\circ}$ & $1^{\circ} \pm 1^{\circ}$ & $<0.001 *$ & $\begin{array}{l}\text { Junior/senior: } \\
\text { Junior/attending: } \\
\text { Senior/attending: }\end{array}$ & $\begin{array}{l}0.05^{*} \\
0.001^{*} \\
0.76\end{array}$ \\
\hline Lateral & $27^{\circ} \pm 7^{\circ}$ & $11^{\circ} \pm 4^{\circ}$ & $7^{\circ} \pm 5^{\circ}$ & $<0.001^{*}$ & $\begin{array}{l}\text { Junior/senior: } \\
\text { Junior/attending: } \\
\text { Senior/attending: }\end{array}$ & $\begin{array}{l}0.001^{*} \\
0.00^{*} \\
0.59\end{array}$ \\
\hline \multicolumn{7}{|c|}{ Residual displacement (percent) } \\
\hline $\mathrm{AP}$ & $7 \% \pm 5 \%$ & $2 \% \pm 1 \%$ & $2.7 \% \pm 1 \%$ & $0.04 *$ & $\begin{array}{l}\text { Junior/senior: } \\
\text { Junior/attending: } \\
\text { Senior/attending: }\end{array}$ & $\begin{array}{l}0.03 * \\
0.03 * \\
0.88\end{array}$ \\
\hline Lateral & $18 \% \pm 14 \%$ & $4 \% \pm 1 \%$ & $4 \% \pm 2 \%$ & $0.01 *$ & $\begin{array}{l}\text { Junior/senior: } \\
\text { Junior/attending: } \\
\text { Senior/attending: }\end{array}$ & $\begin{array}{l}0.05 * \\
0.01 * \\
0.98\end{array}$ \\
\hline
\end{tabular}

Values are mean $\pm \mathrm{SD} ; * \mathrm{p}<0.05$

tissue can lower the fidelity of the simulation causing poor discriminative abilities between individuals of different experience levels $[9,13,15]$. When soft tissues are incorporated in the model to increase haptic feedback, the outer shell of the model makes it difficult to accurately assess the adequacy of the reduction. Thus, although these models can provide biofeedback to develop a feel for the process of fracture reduction, they cannot truly simulate the process of fracture reduction and casting. We thus sought to create a model that requires the learner to master all of the steps of the process to achieve a good final result. When combined with fluoroscopy, the fracture reduction model described here can simulate the entire process of distal radius fracture reduction and the learner can receive haptic and visual feedback on their reduction and casting performance in real time. The quantitative nature of the radiopaque monofilaments at the end of the distal radius fracture also allows for accurate and reproducible measurement of residual displacement and angulation at the fracture site, potentially allowing for its use as an assessment tool. In addition to the noted educational value reported by the resident and attending surgeon participants, the current study showed that the performance metrics are reliable and valid when stratified across level of training and expertise.

This study should be viewed in the context of its limitations. Although the model simulates shoulder motion in three planes, the elbow is a hinged articulation not allowing participants to take into account forearm rotation during fracture reduction. Furthermore, a simple transverse extraarticular fracture was simulated in the model, thereby affecting the participant's ability to learn the nuances required to reduce and cast different fracture patterns. The simulated periosteum and internal deforming forces of this simple fracture pattern were refined over several prototypes and did not incorporate pretesting cadaveric or biomechanical analysis of the forces required to reduce a distal radius. Future research of the biomechanical analysis of distal radius reduction and how this can be translated to synthetic bone models will help improve simulation training.

In addition, during the assessment, reviewers were not blinded to the participant's year in training. However, objective measures were used with excellent interrater reliability. Residents were not randomly selected to participate in the study, but rather represented a convenience sample of those rotating on the pediatric orthopaedic service. Although this has the potential to introduce selection bias, all testing was done in two consecutive resident rotations thereby eliminating variation in training that would be introduced by testing all residents at different times during the academic year. Similarly, questionnaire information was potentially subjected to bias because study subjects were not blinded to the identity of the investigators. However, because tactile simulation is very subjective, and no validated instruments exist that are able to quantify model "realness," we thought that asking participants' subjective opinions regarding the model would be the best way to understand its "authenticity". In the assessment of the model, there was lack of discrimination between groups regarding the secondary performance variables. These nonsignificant findings may have been secondary to insufficient power. Future research should involve larger sample sizes stratified across various residency programs to help define the educational effect the 
model imparts in training. Finally, fluoroscopy is required to get detailed objective feedback on the model. Although participants can feel the fracture and gauge their reduction clinically, to truly see how much displacement and angulation are left at the fracture site, radiographs are needed and may not be available for training at all sites. The required use of fluoroscopy potentially exposes participants to increased radiation in the short term. However, the goal of the simulator is to decrease participant and patient radiation exposure by minimizing the learning curve. All institutional radiation safety precautions were followed throughout the course of this study, and trained radiology technologists were present to enforce such regulations. Future comparison studies assessing "on-the-job" training versus simulation training will help clarify the effectiveness of this model in residents' learning. Despite these limitations, we believe that the model used for the current study is a large improvement over existing training models and may serve as a basis for further improvements going forward. These are areas of continuing research and design to help provide a fully encompassing simulation experience.

The acquisition of fracture reduction skills has been evaluated by several authors through the use of bench-top table models $[9,15]$. Both studies showed that simulation improves technical clinical skills. Egan et al. [9] developed a bench-top model for distal radius fracture treatment with the primary objective to assess how much the simulation resembles the situation in the clinical setting. A five-point Likert scale was used to assess the participant's opinion regarding the realism of the model. The study consisted of 55 total participants with a wide range of levels of training. The reported success rate in obtaining a reduction was $93 \%$, and $64 \%$ of participants felt that the simulator reflected real-life fracture reduction scenarios. The limitations of their model surrounded the lack of formal assessment of participant performance and accuracy of the reduction. To assess the reduction, a zipper on the volar aspect of the fracture needed to be opened which increased the risk for loss of reduction.

Mayne et al. [15] improved on this bench-top model by imbedding two intramedullary stainless steel rods in the radius and ulna proximal to the osteotomy site, and two ball markers in the distal radial fragment. Radiographs were taken after the reduction to assess palmar tilt, and a formative assessment of the residents' fracture reduction and cast application was performed through Objective Structured Assessment of Technical Skills and global rating scale [16]. Ninety-five percent of the residents were able to successfully reduce the fracture. In their model, palmar tilt was the only radiographic parameter that could be reliably measured, and was not found to be significantly different between junior and senior residents. In addition, there was no correlation between reduction adequacy and the Objective Structured Assessment of Technical Skills and global rating scale scores. They theorized that their lack of discrimination between year of training may have been secondary to the lack of difficulty with the fracture reduction model.

Our model attempts to increase the difficulty of the simulation by requiring the participant to navigate the difficulties of manipulating an entire mobile extremity during the fracture reduction. Similar to the other models, the participant is required to use proper reduction principles and to apply a cast while maintaining this reduction. If an improper technique is used during fracture reduction or cast molding, the tension of the simulated periosteum will malreduce the fracture, which will be evident either clinically or during radiographic examination. Unlike previous models, our model requires the participant to have the forethought of fracture reduction setup, C-arm positioning, and available cast supplies mimicking real-life scenarios. The resident can practice long and short arm casting. In either circumstance, they need to control the entire upper extremity limb during cast application. This can be done through finger traps or an assistant. This is in contrast to available fracture reduction and casting models that use a tabletop clamp to secure the forearm during the procedure.

In addition to the fracture reduction and casting considerations, the simulated elbow and shoulder motion planes imitate real-life issues that can arise while trying to incorporate imaging in the reduction. To obtain appropriate imaging during the simulation, the resident needs to position the arm in the appropriate plane or maneuver the fluoroscopy machine to obtain the correct images. Improper positioning leads to malrotated views requiring further fluoroscopy and increased radiation exposure to the patient and personnel in the room. It also greatly increases the time required to complete the task if the learner has not anticipated these challenges. Learning to master the subtle techniques of obtaining the appropriate imaging will decrease fluoroscopy use and radiation exposure during these manipulations when performed in real-life situations.

The Accreditation Council for Graduate Medical Education has highlighted distal radius fracture reduction as one of the essential concepts to be learned during the first year of orthopaedic residency [8, 11]. Similarly, the American College of Emergency Room Physicians has made distal radius fracture reduction an important procedure in their learning curriculum [5, 6]. An education curriculum built around this simulation model may hasten the learning curve and improve patient outcomes early on during the junior residents' training.

We described the development of a fracture reduction simulator for which performance metrics are reliable and valid when stratified across level of training and expertise. 
The model requires the participant to use proper reduction and cast molding principles while also realistically manipulating the shoulders and elbows to obtain the appropriate images. Future directions will include refining the model to incorporate more-difficult fracture patterns, and developing models that are capable of capturing the accuracy of the reduction that are not reliant on radiographic feedback, but not at the price of the simulation. The goal is to optimize resident education before patient interactions. A fracture reduction and casting curriculum built around this model may maximize learning opportunities while minimizing patient risk.

Acknowledgments We thank Thom Porro and all of the wonderful engineers at Sawbones ${ }^{\circledR}$ for helping to bring this idea to life.

\section{References}

1. Akhtar KS, Chen A, Standfield NJ, Gupte CM. The role of simulation in developing surgical skills. Curr Rev Musculoskelet Med. 2014;7:155-160.

2. Alemdaroglu KB, Iltar S, Cimen O, Uysal M, Alagoz E, Atlihan D. Risk factors in redisplacement of distal radial fractures in children. J Bone Joint Surg Am. 2008;90:1224-1230.

3. Asadollahi S, Ooi KS, Hau RC. Distal radial fractures in children: risk factors for redisplacement following closed reductions. $J$ Pediatr Orthop. 2015;35:224-228.

4. Bae DS. Pediatric distal radius and forearm fractures. $J$ Hand Surg Am. 2008;33:1911-1923.

5. Bank I, Cheng A, McLeod P, Bhanji F. Determining content for a simulation-based curriculum in pediatric emergency medicine: results from a national Delphi process. CJEM. 2015;17: 662-669.

6. Chapman DM, Hayden S, Sanders AB, Binder LS, Chinnis A, Corrigan K, LaDuca T, Dyne P, Perina DG, Smith-Coggins R, Sulton L, Swing S. Integrating the Accreditation Council for Graduate Medical Education Core competencies into the model of the clinical practice of emergency medicine. Acad Emerg Med. 2004;43:756-769.

7. Chikwe J, de Souza AC, Pepper JR. No time to train the surgeons. BMJ. 2004;328:418-419.

8. Dougherty PJ, Marcus RE. ACGME and ABOS changes for the orthopaedic surgery PGY-1 (intern) year. Clin Orthop Relat Res. 2013:471:3412-3416.
9. Egan C, Egan R, Curran P, Bryan K, Fleming P. Development of a model for teaching manipulation of a distal radial fracture. $J$ Bone Joint Surg Am. 2013;95:433-438.

10. Fabricant PD, Dy CJ, Dare DM, Bostrom MP. A narrative review of surgical resident duty hour limits: where do we go from here? $J$ Grad Med Educ. 2013;5:19-24.

11. Gardner AK., Scott DJ, Hebert JC, Mellinger JD, Frey-Vogel A, Ten Eyck RP, Davis BR, Sillin LF, Sachdeva AK. Gearing up for milestones in surgery: will simulation play a role? Surgery, 2015; 158:1421-1427.

12. Haddad FS, Williams RL. Forearm fractures in children: avoiding redisplacement. Injury. 1995;26:691-692.

13. Ho K, Chimutengwende-Gordon M, Hardy JR. A simple model to demonstrate the method of reduction and immobilization of forearm fracture in an adult or child. Ann $R$ Coll Surg Engl. 2006;88:224-225.

14. Jones K, Weiner DS. The management of forearm fractures in children: a plea for conservatism. $J$ Pediatr Orthop. 1999;19:811-815.

15. Mayne IP, Brydges R, Moktar J, Murnaghan ML. Development and assessment of a distal radial fracture model as a clinical teaching tool. J Bone Joint Surg Am. 2016:98:410-416.

16. Moktar J, Popkin CA, Howard A, Murnaghan ML. Development of a cast application simulator and evaluation of objective measures of performance. J Bone Joint Surg Am. 2014:96:e76.

17. Pershad J, Williams S, Wan J, Sawyer JR. Pediatric distal radial fractures treated by emergency physicians. J Emerg Med. 2009;37:341-344.

18. Pretell Mazzini J, Rodriguez Martin J. Paediatric forearm and distal radius fractures: risk factors and re-displacement: role of casting indices. Int Orthop. 2010;34:407-412.

19. Proctor MT, Moore DJ, Paterson JM. Redisplacement after manipulation of distal radial fractures in children. $J$ Bone Joint Surg Br. 1993;75:453-454.

20. Rodriguez-Merchan EC. Pediatric fractures of the forearm. Clin Orthop Relat Res. 2005;432:65-72.

21. Roy DR. Completely displaced distal radius fractures with intact ulnas in children. Orthopedics. 1989;12:1089-1092.

22. Ryan LM, Teach SJ, Searcy K, Singer SA, Wood R, Wright JL, Chamberlain JM. Epidemiology of pediatric forearm fractures in Washington, DC. J Trauma. 2010;69(4 suppl): S200-205.

23. Scott DJ, Cendan JC, Pugh CM, Minter RM, Dunnington GL, Kozar RA. The changing face of surgical education: simulation as the new paradigm. J Surg Res. 2008;147:189-193.

24. Sharma S, Bowe D, Walters SJ, Flowers MJ. Dorsal cortical comminution as a predictor of redisplacement of distal radius fractures in children. Injury. 2011;42:173-177.

25. Voto SJ, Weiner DS, Leighley B. Redisplacement after closed reduction of forearm fractures in children. J Pediatr Orthop. 1990;10:79-84. 